\title{
Algunos problemas en torno a la noción de sánscrito clásico*
}

\author{
Gabriela Müller ${ }^{1}$ \\ Universidad Buenos Aires (Argentina) \\ Verónica Orqueda ${ }^{2}$ \\ Pontificia Universidad Católica de Chile (Chile)
}

Recibido: octubre 30 de 2015. Revisado: enero 25 de 2016. Aceptado: marzo 20 de 2016

Referencia norma APA: Müller, G. \& Orqueda, V. (2016). Algunos problemas en torno a la noción de sánscrito clásico. Rev. Guillermo de Ockham, 14(1), 107-114. doi: http://dx.doi.org/10.21500/22563202.2311

\section{Resumen}

En este trabajo reflexionamos sobre algunos problemas en torno a la noción de sánscrito en general y de sánscrito clásico en particular. Partiendo de una serie de distinciones, discutimos cuatro caracterizaciones diferentes, pero relacionadas entre sí, del término "sánscrito": (a) en cuanto lengua clásica; (b) en cuanto variedad lingüística natural; (c) en cuanto lengua en la que está compuesto el canon literario de la India antigua; y (d) en tanto lengua artificial. Estas caracterizaciones, junto con los problemas asociados a ellas, llevan directamente a considerar los desafíos que entraña la enseñanza de esta lengua, así como las posibles líneas de investigación que deben ser profundizadas.

Palabras clave: sánscrito, lenguas clásicas, variedad lingüística, tradición gramatical sánscrita, literatura de la India antigua

\section{Some issues concerning the notion of Classical Sanskrit}

\section{Abstract}

This paper addresses some problems related to the question about what is Sanskrit in general, and what is Classical Sanskrit in particular. After offering some of the best known depictions of the language, we establish four different (but related) characterizations that lie beneath the ways we use the notion of "Sanskrit": (a) as a classical language, (b) as a natural linguistic variety, (c) as the language of the ancient Indian literary canon, and (d) as an artificial language. The problems and limitations of the diverse criteria involved in each of these categorizations will lead us to draw some final considerations regarding the peculiarities of the Sanskrit language, the challenges entailed by its learning process and some possible lines for further research.

Keywords: Sanskrit, classical languages, linguistic variety, Sanskrit grammatical tradition, ancient Indian literature

* Este trabajo es una versión ampliada de una comunicación presentada en el simposio "Enseńanza y traducción del sánscrito en el mundo de habla hispana”, que tuvo lugar en el marco del XV Congreso Internacional Aladaa, celebrado en Santiago de Chile. Agradecemos a los coordinadores y asistentes a dicho simposio por sus aportes y comentarios.

1. Doctora en Filosofía por la Universidad de Buenos Aires. Docente auxiliar de las cátedras de Sánscrito (Departamento de Lenguas y Literaturas Clásicas) y de Historia de la Filosofía Antigua (Departamento de Filosofía) en la Facultad de Filosofía y Letras de la Universidad de Buenos Aires. Becaria postdoctoral del Consejo Nacional de Investigaciones Científicas y Técnicas (Conicet). Miembro de los proyectos de investigación 20020130100022BA (UBACyT) y PICT (FONCyT) 2013-0588, 2014-2017. Correo electrónico: gafermu@gmail.com. Dirección postal: Puán 480, $4^{\circ}$ piso, oficina 435, Ciudad Autónoma de Buenos Aires (1460), Argentina.

2. Doctora en Estudios del Mundo Antiguo por la Universidad Complutense de Madrid. Docente del Departamento de Ciencias del Lenguaje, Facultad de Letras, Pontificia Universidad Católica de Chile. IR del proyecto CONICYT-FONDECYT 3150246, 2014-2017 (Chile) y miembro del proyecto FFI2012-36069-C03-02, 2013-2015, del Ministerio de Economía y Competitividad (España).Correo electrónico: vorqueda@uc.cl. Dirección postal: Av. Vicuńa Mackenna 4860, Macul, Región Metropolitana, Chile. 


\section{Alguns problemas em torno da noção de sânscrito clásico}

\section{Resumo}

Neste trabalho vamos refletir sobre alguns problemas que surgem quando se tenta responder a pergunta que é o sânscrito, em geral, e que chama-se sânscrito clássico, em particular. Vamos apontar quatro caracterizações diferentes, relacionados, que fundamentam nosso uso do termo "sânscrito" (a ) tanto em linguagem clássica, (b) como uma variedade natural, linguística, (c) como a língua em que é feito o cânone literário da Î́ndia antiga e (d) como linguagem artificial. Os problemas e as limitaçóes dos vários critérios envolvidos em cada uma dessas caracterizaçóes nos levará a algumas reflexões finais sobre as particularidades do idioma sânscrito, os desafios do ensino e possíveis linhas de pesquisa que devem ser aprofundadas.

Palavras-chave: Sânscrito, línguas clássicas, a variedade linguística, sânscrito gramatical

\section{Introducción: sánscrito y sánscrito clásico}

\author{
As a deliberately standardized and maintained \\ yet flexible medium of elite communication Sanskrit is \\ a fascinating product of the human spirit \\ (Masica, 1991, p. 6).
}

En términos generales, se suele hablar de "sánscrito" en sentido amplio y en sentido estricto. En el primer caso, se lo considera como sinónimo de indoario antiguo, es decir, el estadio más antiguo de las lenguas indoarias, pertenecientes a la rama indoirania de la familia de lenguas indoeuropeas. Según este sentido amplio, el sánscrito abarcaría tanto la variedad preclásica (védico) como la clásica (sánscrito clásico) y se opondría, en cambio, al prácrito, en tanto sinónimo de indoario medio. Por otro lado, en un sentido más estricto, sánscrito puede referir a la lengua clásica de manera independiente, diferente del védico preclásico. Según este segundo sentido, la mayoría de los estudiosos señalan que el sánscrito es la lengua codificada en la Aștāidhyāyì de Pānini y que permanece invariable desde entonces. ${ }^{3}$ Sin embargo, esto no impide que a menudo se señalen rasgos diferenciales dentro de las obras compuestas en sánscrito que hacen necesario al menos matizar esta supuesta inmutabilidad. Así, por ejemplo, se diferencia entre el sánscrito épico (la lengua en la que están compuestos el Mahābhärata y el Rämāyaṇa) que suele no seguir ciertas reglas establecidas por Pānini, el sánscrito puránico y el sánscrito de la literatura clásica o directamente sánscrito clásico (Burrow, 1955; Mylius, 2015 y Aklujkar, 1996).

A partir de tales variaciones, sería posible identificar el sánscrito clásico con la lengua de las grandes obras del período clásico (alrededor de los siglos IV y V d.C.) y diferenciarlo respecto de la lengua fijada por Pānini, la cual podría fecharse dentro del período védico tardío y que se distingue del védico temprano (el lenguaje de los cuatro veda), proyectando así la división en períodos que suele hacerse de la historia de la India antigua en la distinción de las variantes lingüísticas de los textos supuestamente compuestos en dichos períodos (Cardona, 1987).

En este trabajo nos proponemos mostrar qué implicancias tiene entender el sánscrito de un modo u otro; es decir, según se tome en cuenta un criterio lingüístico, textual, histórico o cultural. Para esto estableceremos una distinción cuádruple que pretende dar cuenta de una gama de sentidos diversos, aunque relacionados, que están detrás del término "sánscrito": a. en tanto lengua clásica; b. en tanto variedad lingüística natural; c. en tanto lengua en la que está compuesto el canon literario de la India antigua; $\mathrm{y} d$. en tanto lengua artificial.

\section{El sánscrito como lengua clásica, equivalente al griego y el latín}

En este primer sentido, la denominación de clásico aplicada al sánscrito sigue un criterio fundamentalmente histórico que se explica por la asociación con las lenguas hoy consideradas clásicas: específicamente, el griego y el latín.

Ahora bien, la delimitación de los períodos llamados clásicos de diferentes civilizaciones responde al reconocimiento de cierto auge cultural anclado en un período sociohistórico determinado y reflejado en las variantes lingüísticas en las que fueron compuestos sus textos. Así,

3. "With Panini's work Sanskrit in its external form became finally stabilised and no more change was allowed. From then on the history of Indo-Aryan is the history of Middle Indo-Aryan in its various phases (Pali, Prakrit, Apabhramśa), and then Modern Indo-Aryan. In this evolution Sanskrit took no part, but remained as it was fixed by Panini at a period long antedating the bulk of the classical literature" (Burrow, 1955, p. 36). 
el griego clásico corresponde al período comprendido entre los siglos v y iv a.C. aproximadamente, en estrecha relación con el esplendor ateniense y con el auge de la filosofía y la literatura, gracias a figuras como las de Platón y Aristóteles en filosofía y Esquilo, Sófocles, Eurípides y Aristófanes en el drama.

Dado que el dialecto predominante en este período de Atenas era el ático, el griego clásico está asociado a esta variedad lingüística (Berenguer-Amenós, 1999), a pesar de que por entonces en el mundo helénico ya existía una amplia gama de variedades, muchas de ellas distribuidas regionalmente. De esta manera, el denominado griego clásico excluye textos clave de la Grecia antigua como lo son las dos grandes epopeyas atribuidas a Homero, principalmente desde un punto de vista temporal, aunque también desde un punto de vista sociolingüístico, debido a la presencia de una importante mezcla de variedades lingüísticas, por un lado, y al diferente contexto de elaboración de estas obras con respecto a aquellas clásicas. Por otro lado, también se pueden excluir de este período otras obras y variedades de períodos posteriores, como por ejemplo aquellas en las que se utiliza la denominada koiné, un dialecto fundamentalmente literario, y que muchas veces no era el dialecto materno del autor.

Por su parte, el latín clásico puede fecharse entre los siglos I a.C. y I d.C., es decir durante la República de Augusto, cuando se propició el auge de la literatura y la cultura con autores como Ovidio, Virgilio y Cicerón. De esta manera, el denominado latín clásico se diferencia, en primer lugar, del arcaico (siglos III a II a.C.), la variedad de autores como Plauto, Terencio, Ennio o Nevio, que conservaría rasgos más cercanos a una protolengua, y, en segundo lugar, del latín posclásico propio del Imperio (variedad que correspondería a la lengua de autores como Séneca y Apuleyo), cuando se producen importantes cambios socioculturales y la decadencia de varios géneros literarios.

Según este criterio, la categoría de clásico en el contexto de la India antigua debería corresponder al período de auge cultural ocurrido durante la dinastía Gupta, alrededor de los siglos iv y v d.C. Así, los textos sánscritos compuestos durante esa época, como los de Kālidāsa y Bhartṛhari, podrían denominarse indiscutiblemente clásicos. Pero al mismo tiempo, la aplicación de este criterio llevaría a dudar respecto de la clasificación de otras composiciones, como por ejemplo textos épicos u obras científicas o filosóficas que a menudo son consideradas clásicas y cuyo período de composición, sin embargo, no puede limitarse a este período temporal, en tanto fueron compuestas a lo largo de períodos temporales más extensos, a veces de varios siglos. Incluso la gramática de Pānini puesto que se trataría de una obra al menos ocho siglos anterior a tal período.

Por otro lado, la categoría de clásico aplicada al sánscrito a partir del modelo del griego y el latín responde al hecho de que los europeos se sirvieron de estas otras lenguas ya conocidas para la elaboración de las primeras gramáticas durante los siglos XVII y XVIII, lo cual se evidencia, por ejemplo, en la terminología utilizada. ${ }^{4} \mathrm{Sin}$ duda, el impulso propiciado por la lingüística comparativa indoeuropea durante el siglo XIX termina de delinear el paralelo entre estas tres lenguas.

Por último, se podrían relacionar los casos del sánscrito y del latín en la actualidad, en la medida en que parecería haber un intento de revitalización de ambas lenguas. Sin embargo, la situación no es completamente similar puesto que, en el caso del latín, se intenta la recuperación de una lengua que continúa considerándose antigua, mientras que en el caso del sánscrito se apunta a la pervivencia de una lengua vigente en el marco de un proyecto nacionalista. Nótese, por ejemplo, que el sánscrito es una de las tantas lenguas oficiales de la República de la India y la segunda lengua oficial del estado de Uttarakhand, en el norte de este país; en el censo del año 2001, 14.135 personas reportaron el sánscrito como su lengua nativa; desde 1947 se han compuesto más de 3.000 obras literarias en esta lengua y, especialmente en las últimas décadas, desde distintos sectores del gobierno indio, se han hecho esfuerzos por incentivar su uso.

\section{El sánscrito como variedad lingüística natural}

Dada la complejidad que entraña la distinción entre dialecto y lengua (Masica, 1991), optaremos por utilizar el concepto de variedad lingüística, a pesar de su posible vaguedad, para hacer referencia a una forma determinada de una lengua, caracterizada por la presencia de un conjunto específico de elementos lingüísticos. ${ }^{5}$

La caracterización antes vista del sánscrito como una lengua clásica conlleva una diferenciación respecto del védico, en cuanto lengua preclásica. Si esta distinción

4. Por ejemplo, en la gramática de Hanxleden (ca. 1712-1732), editada recientemente por Toon Van Hall y Christopher Vielle (2013), se distinguen tres ablativos en el sistema nominal, de los cuales dos corresponden a casos de los que carece el latín clásico y que más tarde se denominarán "instrumental" y "locativo".

5. "What makes one variety of language different from another is the linguistic items that it includes, so we may define a variety of language as a set of linguistic items with similar social distribution" (Hudson, 1996: 22). 
se asocia a dos variedades lingüísticas naturales, es decir, utilizadas por hablantes reales, una primera opción es entender esta variación como diacrónica. $Y$, en efecto, las gramáticas occidentales señalan una serie de diferencias entre ambas. Por ejemplo, en el sistema verbal, hay cierta tendencia hacia la simplificación de formas coexistentes; así, desaparecen desinencias activas como -masi ( $1^{a}$ pl. ind., pres.), -thana ( $2^{\mathrm{a}} \mathrm{pl}$. ind., pres.), -tana ( $2^{\mathrm{a}} \mathrm{pl}$. imperf.), $-d h i$ ( $2^{a}$ sg. imperat.) y $-\bar{a}\left(1^{a}\right.$ sg. subj. pres.), que sí se pueden encontrar en los veda, mientras que otras formas son comunes a ambos tipos de textos, como por ejemplo las desinencias - mah ( $1^{\mathrm{a}} \mathrm{pl}$. ind., pres.), -tha ( $\mathrm{a}^{\mathrm{a}}$ pl. ind., pres.), -ta ( $2^{\mathrm{a}} \mathrm{pl}$. imperf.), - $h i$ ( $2^{\mathrm{a}}$ sg. imperat.)

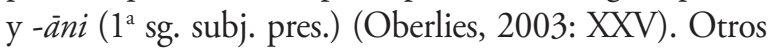
rasgos conocidos que no habrían estado presentes en los textos védicos pero sí en textos posteriores y que vale la pena mencionar son, en el campo fonético/fonológico, la desaparición de la vocal /I!l, en el ámbito morfológico, la caída del uso de los modos subjuntivo e injuntivo, y en el ámbito sintáctico y estilístico, la exacerbada utilización de compuestos nominales muy extensos, cuyo desarrollo en una variedad natural parece bastante improbable.

Consideradas estas dos variedades como naturales, también podrían diferenciarse entre sí según un criterio geográfico, puesto que el védico está basado en una variedad del extremo oeste (quizás influenciada por variedades iranias, al menos en algunos libros del Rgveda), mientras que el sánscrito clásico se habría conformado a partir de variedades de la región central: la región occidental del valle del Ganges, la región este de Punjab y Haryana (Masica, 1991: 51).

Ahora bien, lo más corriente, cuando se señala la contraposición entre el sánscrito y el védico como variantes naturales, es identificar al primero con la lengua sistematizada por Pāṇini. Sin duda, la variedad Așțādhyāyì se distancia de lengua los veda en varios aspectos. Así, por ejemplo, Pāninin no distingue semánticamente entre tres tipos de pasado (imperfecto, perfecto y aoristo), que sí se habrían usado con significados diferentes en los brähmana y las upanișad, y por eso el aoristo para él habría servido de modo general para el pasado, mientras que en los otros dos tipos de textos se habría limitado al pasado reciente (Deshpande, 2001); en segundo lugar, Pānini utiliza el término upasarga para las partículas como pra cuando aparecen con verbos, es decir, las considera como "adiciones" o algo que se adjunta a otro elemento: upasargāh kriyāyoge (1.4.59). ${ }^{6}$ Sin embargo, el papel que desempeñan estas partículas en textos como el Rgveda es mucho más libre y más cercano quizás a lo que hoy consideraríamos un adverbio o un modalizador y, por lo tanto, en védico no correspondería hablar de 'anexo', tal como en la denominación de Pāninini (Deshpande, 1996). Por otra parte, este camino histórico, desde una posición más libre hacia una más ligada, tal como se ve en este caso, es bastante frecuente en el desarrollo de las lenguas indoeuropeas antiguas.

No obstante, la mera distinción entre sánscrito y védico podría resultar demasiado simplista a la hora de caracterizar las diferentes variedades naturales, puesto que hay textos, como el Atharvaveda, cuya datación es mucho más compleja y cuya lengua presenta simultáneamente tanto un conjunto de rasgos muy arcaizantes como importantes innovaciones (Kulikov, 2013). Este punto pone de manifiesto precisamente el problema que entraña trazar distinciones taxativas ya que es posible detectar variaciones diacrónicas dentro del grupo de obras que están compuestas en védico, no solo entre lo que suele denominarse védico temprano (la lengua de los cuatro veda) y védico tardío (la lengua de los brähmaṇa, las upanișad más antiguas y ciertos sütra tempranos) (Cardona, 1987: 20-27), sino también dentro del primer grupo (el védico temprano) cuyo estadio más antiguo sería el que aparece en los himnos de los libros II a VII del Rgveda (Witzel, 1989).

Así, señalar un punto más o menos claro de delimitación entre védico y sánscrito es doblemente complejo: en primer lugar, porque el cambio lingüístico nunca se da por categorías taxonómicas, sino que se trata más bien de un continuo constante y progresivo; y en segundo lugar, porque eso implica una toma de posición con respecto a la mutabilidad o inmutabilidad de la lengua por parte de los gramáticos indios. En efecto, se suele señalar que estos no desarrollaron una teoría del cambio diacrónico, sino que veían al sánscrito como una lengua eterna, permanente e invariable (Deshpande, 2001); sin embargo, esto no les habría impedido reconocer, en su descripción de esta lengua, la diversidad lingüística real que percibían, aunque no lo hayan admitido abiertamente. De esta manera, ciertos factores que consideramos históricos estarían representados dentro de un paradigma ahistórico: el sánscrito, como lengua eterna, está más allá de la noción de cambio, pero la información disponible sobre esa lengua, por el contrario, va cambiando con las sucesivas generaciones que la estudian, de modo que el conocimiento que los gramáticos tienen de ella siempre es pasible de ser aumentado y perfeccionado (Deshpande, 1993). De hecho, si nos atenemos a lo seńalado explícitamente por

6. Aquí y en lo sucesivo, las citas de la Aștāidhyāyĩ de Pāṇini están tomadas del texto de Cardona (1988). 
Pānini, lo cierto es que en varias partes de su obra aparece una contraposición entre bhāșa (que podría ser asociada con la lengua hablada) y chandas (que podría ser asociado a la lengua sagrada en la que están compuestos los veda). También aparecen mencionadas algunas diferencias entre las variantes lingüísticas que se encuentran "en el norte" (udīcām) y "en el este" (prācām). ${ }^{7}$ Estas distinciones sirven para explicar irregularidades de determinadas reglas propuestas, que se pueden restringir a una variable u otra o al cruce de ambas. Así, por ejemplo, los sütra 6.3 .32 y 33 indican que un mismo compuesto dvandva se realiza de manera diferente "en la lengua sagrada" (chandasi) y "en el norte" (udīcām): mätarapitarāv udīcām (6.3.32) / pitarāmātarā ca cchandasi (6.3.33). ${ }^{8}$

Ahora bien, la mención de tales diferencias no debe interpretarse necesariamente como una referencia a dos variedades naturales diferentes. En particular, en el caso de la distinción entre bhāṣā y chandas, no hay por qué inferir sin más que se trata de una distinción anclada en criterios geográficos o temporales, ya que podría tratarse, por ejemplo, de una variación de tipo textual o discursiva: la que se encuentra "en los textos sagrados" (chandasi) por contraposición a aquella que se verifica "en el habla" (bhāșāyām). ' A este tema nos referiremos precisamente en la siguiente sección.

\section{El sánscrito como lengua del canon literario}

La concepción del sánscrito como lengua clásica, vista en la primera sección, está íntimamente relacionada con su identificación con la lengua en la que está compuesto el canon literario de la India antigua. En tal sentido, se lo entiende no ya como una variedad lingüística natural (como en la sección b), sino como la lengua de un conjunto de textos que se ha ido conformando a lo largo de los siglos. Según esta acepción, el sánscrito ya no se diferenciaría del védico (y tampoco de los prácritos, a los que aludiremos enseguida) por un criterio cronológico, sino más bien por uno discursivo-textual, lo cual implica considerar también la finalidad de composición de los textos. Es evidente, por ejemplo, que la opacidad de lengua de los veda se relaciona con su función de recitación en contextos rituales y no necesariamente con un fin artístico y literario. Ahora bien, los criterios cronológico y discursivo-textual se pueden combinar si consideramos que el sánscrito, en tanto lengua de la literatura y asociado a valores estéticos, es un desarrollo posterior de una lengua restringida en un primer momento al ámbito ritual y religioso. Nuevamente esta podría ser también la manera de interpretar la contraposición entre chandas y bhāṣā que aparece en Pāṇini.

Desde este punto de vista, entonces, es posible considerar al sánscrito como la lengua estándar de un conjunto de textos compuestos en contextos socioculturales diversos, y fundamentalmente como herramienta de producción literaria y de divulgación cultural. Según esta perspectiva, incluso autores que habrían tenido como lengua materna no el sánscrito sino algún prácrito se habrían servido de este para la composición de textos de valor literario, es decir textos caracterizados por ciertos rasgos del lenguaje que no eran propios de las variantes orales naturales.

Una muestra de la compleja relación entre el sánscrito y las lenguas vernáculas puede verse en ciertos autores budistas. Aśvaghoșa, por ejemplo, utilizaba el sánscrito en determinados textos, aun no siendo su lengua materna, mientras que en otras obras mantenía el $p \bar{a} l i$, el prácrito asociado a la literatura del canon budista (Mylius, 2015).

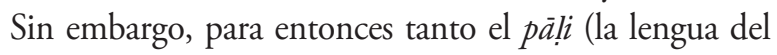
budismo primitivo) cuanto el ardhamägadhì (la lengua del jainismo) habían dejado de ser variedades naturales y convertido en lenguas estandarizadas, al igual que el sánscrito. Ahora bien, muchos autores budistas posteriores comenzaron a "resanscritizar" sus textos; es decir, a trascribirlos observando las reglas fonéticas del sánscrito, dando lugar al nacimiento de una variedad nueva e híbrida (Mylius, 2015), mientras que otros utilizaron directamente el sánscrito para la composición de sus textos, como por ejemplo Nāgārjuna.

Sin duda, la adopción del criterio discursivo-textual pone en evidencia lo difícil que resulta determinar qué es literatura y qué no lo es. ${ }^{10}$ Así, por ejemplo, un pasaje del Śrngazraprakāśa de Bhoja realiza una clasificación de los tipos de discurso (väkyam) y agrupa, dentro de los que

7. El locativo chandasi se registra más de una centena de veces en la Aștāadhyāyzin, mientras que el locativo bhāṣāyām se registra solo siete veces. Por otra parte, el locativo prācām aparece en dieciocho ocasiones, mientras que udìcām aparece solo siete veces.

8. No solo se marca la diferencia en cuanto al orden de los elementos, sino también la posible desinencia - $\bar{a}$ del dual, típica del védico, "more tan seven times as common as that in -au in the Rgveda" (Macdonell, 1916).

9. En efecto, el hecho de que chandas, el estudio del metro, sea uno de los seis vedãnga, permite su asociación más con la aplicación de una serie de reglas que con un tipo de variedad lingüística. Nótese, además, que este término se relaciona con el verbo chad- 'agradar', 'parecer bueno', lo cual pone en evidencia la importancia para su definición del efecto que algo es capaz de producir. En cambio, la relación etimológica entre bhāșā y la raíz verbal bhāṣ- 'decir', 'hablar', 'nombrar' permite pensar, por ejemplo, en el tipo diferente de reglas que se aplicarán.

10. "There are good reasons for arguing - and many have argued this for the past two decades or more- that anything can be literature; that the term needs to be understood pragmatically rather than ontologically as pointing to ways certain texts are used rather than defining what those texts inherently and essentially are" (Pollock, 2003, p. 2). 
corresponden al sánscrito, textos muy diferentes entre sí (mantra, brähmana, smriti, purāna, kävya y śästra), pero que de manera conjunta se diferenciarían fundamentalmente de los otros dos tipos de discurso: präkrta (las lenguas "naturales") y apabhramśa (las lenguas "decadentes" o "corruptas") (Pollock, 2003).

Entre las obras que suelen considerarse indiscutiblemente propias del canon literario, encontramos algunas del período clásico, como las de Kālidāsa y Bhartṛhari, pero también otras anteriores, como los textos épicos y varias obras pertenecientes a la literatura puránica. Ahora bien, la restricción del sánscrito a la lengua de este corpus textual implica la exclusión de un número de obras que no compartirían tal vez ciertos rasgos de estilo ni finalidades, aunque desde otros puntos de vista también se considerasen compuestas en sánscrito. Sin duda, es problemático decidir qué autores y obras se incluyen en este canon literario y cuáles no, e incluso si es posible hablar de un único canon de textos sánscritos.

\section{El sánscrito como variedad artificial}

En estrecha relación con el punto anterior, el propio nombre samskrta da cuenta de la concepción de esta lengua como perfecta, purificada, depurada. Desde esta nueva perspectiva, la categoría de lengua natural quedaría restringida a los diferentes prácritos, ${ }^{11}$ mientras que el sánscrito se definiría por su carácter artificial, adquirido y utilizado por parte de una comunidad muy restringida y con fines específicos; una variedad culta y refinada, asociada a la clase social dominante (encarnada en la casta brahmánica) y especialmente vinculada a la cultura del poderoso y al conjunto de ideologías sostenidas por él (Aklujkar, 1996).

En este punto, es interesante señalar que, al menos en un principio, las lenguas de las obras surgidas al interior del budismo y el jainismo (movimientos que surgieron por oposición a esta ideología dominante) fueron precisamente vernáculas: pāli y ardhamāgadhì. Del mismo modo, en las obras dramáticas, los personajes de castas más altas hablan en sánscrito, mientras que los pertenecientes a castas más bajas utilizan diferentes prácritos.

El sentido primario del participio saṃskrta- es "compuesto", "preparado", "confeccionado", "adornado", "purificado". Cabe aclarar que, si bien Pāninini explica su formación en los sütra 6.1.137-138 y también lo utiliza como un adjetivo con el sentido de "preparado" (refi- riéndose específicamente a la comida, en los sūtra 4.4 .3 y 4.2.16), jamás usa este término para referirse a la lengua que está sistematizando. Fue con posterioridad que esta palabra comenzó a ser utilizada de este modo, o bien como sustantivo neutro (samskrtam), o bien como adjetivo acompañando a los sustantivos vāc o gir (Cardona, 1988 y Aklujkar, 1996). ${ }^{12}$

Si bien la consideración del sánscrito como una variante lingüística artificial parece estar en las antípodas de su identificación con la lengua fijada por Pānini, que reflejaría el lenguaje hablado en ese momento y lugar, lo cierto es que la labor de los gramáticos -empezando por el propio Pānini- parece haber sido precisamente la de "depurar" esta lengua para hacerla perfecta. Lo que en este punto se evidencia es la dicotomía entre ver la labor de los gramáticos indios como una descripción del lenguaje en uso o como una actividad prescriptiva y, en este sentido, "purificadora" de una lengua que se aleja del lenguaje hablado y que, en consecuencia, termina siendo un artificio.

La misma tensión está en juego en el Mahābhāșya de Patañjali. Si bien para este gramático el sánscrito es aquella lengua sistematizada por Pāninini, al comentar el sūtra prșodarädīni yathopadiștam (6.3.109) en Mahābhäșya III.173-19-174.15, alude a los denominados sișț, hablantes modelo sobre cuya autoridad se basa el uso correcto de la lengua más allá de lo establecido por Pānini (Cardona, 1988).

Parece claro que el sánscrito de esa época ya no era una variedad natural (no era lengua materna de nadie) y además presentaba diferencias con la lengua codificada por Pāninini cuyas formas en algunos casos se habían vuelto obsoletas. Deshpande (1993), por ejemplo, distingue dos usos no paninianos del sánscrito en esa época: uno aceptado por los gramáticos en base a la autoridad de los śișta (que este autor denomina sánscrito "estándar") y otro no aceptado (que el autor denomina sánscrito "vernáculo"); y la mayor o menor influencia de los prácritos sería una de las variables para distinguirlos, ya que ambos registros (sánscrito y prácritos) convivieron a lo largo de los siglos en una situación de diglosia y, por lo tanto, sufrieron inevitablemente una mutua influencia.

Así, junto a la "sanscritización" de los prácritos (que se evidencia sobre todo en los préstamos llamados tatsa$m a$, cuando conservan la forma sánscrita originaria, $\mathrm{y}$ tadbhava, cuando se apartan de esta) es plausible suponer

11. Tal contraposición entre sánscrito y prácrito puede verse en el siguiente pasaje del Nātyaśástra, 17.1: evaṃ tu saṃskṛtam pāthyaṃ mayā proktam samāsatah /präkrttasya tu pāṭhyasya sampravakșyāmi lakșaṇam (citado por Aklujkar, 1996: 70-71).

12. Uno de los primeros textos donde se usa el adjetivo samskṛta- para referirse a una lengua es el Rāmāyaṇa 5.28.17-18: vācam codāharișyāmi mānușim iha saṃskṛtām // yadi vācam pradāsyāmi dvi-jātir iva saṃskṛtām / rāvaṇam manyamānā mām sìtā bhìtà bhavișati.

$112<$ Universidad de San Buenaventura, Cali - Colombia 
también una cierta "pracritización" del sánscrito, que conllevaría diferencias no solo en la pronunciación y en la morfología sino además en el vocabulario. De este modo, el establecimiento de una demarcación taxativa entre sánscrito y prácrito también acarrea dificultades, sobre todo si se tienen en cuenta los "estadios intermedios" o "zonas grises", como el llamado "sánscrito vernáculo", en cuya categoría se incluiría probablemente el conjunto de variedades que en la actualidad muchos hablantes de la India identifican como su lengua materna.

Sin duda, para profundizar en estas cuestiones se requeriría un mayor impulso en los trabajos centrados en dialectología de estas lenguas, que por el momento son escasos y más aún desde una perspectiva histórica.

\section{Conclusiones}

A lo largo de este trabajo, hemos sistematizado en torno a cuatro ejes principales los modos como se puede concebir y problematizar la noción de sánscrito en general y de sánscrito clásico en particular. Como esperamos haber podido mostrar, todos ellos entrañan dificultades, aunque a menudo la vaguedad del término según los distintos criterios analizados se ve asociada a una necesidad de inclusión, más que de exclusión.

De este modo, según un criterio histórico, el sánscrito ha sido equiparado al griego y al latín, aunque las importantes diferencias que existen entre estas lenguas imponen ciertas limitaciones a la pretensión de encontrar también en la India antigua (como en Grecia y en Roma) una lengua clásica. Por otro lado, la consideración del sánscrito como variante natural diferente del védico, es decir tomando en cuenta un criterio fundamentalmente lingüístico, también presenta problemas, ya que parece implicar una delimitación taxativa de categorías excluyentes o discontinuas que difícilmente reflejan las variedades lingüísticas de los diversos textos conservados. En tercer lugar, un criterio discursivo-textual podría ser más incluyente aunque nada fácil de establecer, mientras que un criterio fundamentalmente cultural que considera al sánscrito como un constructo puramente artificial y pretendidamente invariable puede, por último, alejarnos del propio concepto de lengua.

Sin duda, tomar un criterio u otro para definir este complejo sistema lingüístico tiene consecuencias diferentes en el contexto del proceso de enseñanza y aprendizaje, puesto que implica no solo una toma de posición con respecto al conjunto de rasgos gramaticales de la variedad enseñada y a la aceptación de la posible influencia por diglosia de otras variedades, sino también una determinada selección de su literatura y de su cultura en general. En cualquier caso, y más allá del criterio elegido, parece fundamental no excluir la problematización de la noción de sánscrito cuando se enseña esta lengua.

A modo de conclusión, quisiéramos señalar, por último, algunas posibles líneas de investigación que hemos simplemente esbozado en este trabajo y que deberían ser profundizadas para dar una mejor respuesta a la pregunta qué es el sánscrito, esta lengua a la vez flexible y estandarizada; en palabras de Masica (1991) "un producto fascinante del espíritu humano": 1. puntualizar las similitudes y diferencias con el griego y el latín respecto de la diferenciación de sus períodos preclásico, clásico y posclásico y de la relación con las variedades naturales o dialectos; 2. comparar las diferentes clasificaciones en fases existentes para el sánscrito y el védico, que dan cuenta de distintas visiones sobre la diversidad que encierra esta unidad supuestamente invariable; 3 . estudiar los textos de la tradición gramatical india donde se pone de manifiesto su paradigma ahistórico; 4. discriminar los distintos paradigmas mediante los cuales los especialistas occidentales fueron apropiándose del sánscrito para la elaboración de gramáticas y métodos, que son la base utilizada para la enseñanza de esta lengua. Si bien es verdad que hay muchos trabajos sobre estos temas, no lo es menos que todavía queda mucho por hacer.

\section{Referencias}

Aklujkar A. (1996). The early history of Sanskrit as supreme language. In J. Houben (Ed.), Ideology and status of Sanskrit. Contributions to the history of the Sanskrit language (pp. 5985). Leiden: Brill.

Berenguer-Amenós, J. (1999). Gramática griega. Barcelona: Bosch.

Burrow, T. (2001). The Sanskrit Language. Delhi: Motilal Banarsidass.

Cardona, G. (1987). Indo-Aryan Languages. In B. Comrie (Ed.), The Major Languages of South Asia, the Middle East and Africa (pp. 14-79). Londres: Routledge.

Cardona, G. (1988). Pāninini. His Work and its Tradition. Delhi: Motilal Banarsidass.

Deshpande, M. (1996). The Vedic traditions and origins of grammatical thought in Ancient India. In N. Balbir, \& G. J. Pinault (Eds.), Langue, style et structure dans le monde indien: Centenaire de Louis Renou. Actes du Colloque international (pp. 145-169). París: Librairie Honoré Champion. 
Deshpande, M. (1993). Sanskrit and Prakrit. Sociolinguistics Issues. Delhi: Motilal Banarsidass.

Deshpande, M. (2001). The Vedic context of Panini's Grammar. In H. Kniffka (Ed.), Indigenous Grammar across Cultures (pp. 33-51). Frankfurt: Peter Lang.

Hudson, R. A. (1996). Sociolinguistics. Cambridge: Cambridge University Press.

Kulikov, L. (2013). An Atharvanic hymn to night: text-critical and linguistic remarks on the interpretation of Śaunakiya 19.50 = Paippalāda 14.9*. Bulletin of SOAS, 76(2), 259-269. doi:10.1017/S0041977X13000074.

Macdonell, A. (1916). A Vedic Grammar for Students. Oxford: Clarendon Press.

Masica, C. (1991). The Indo-Aryan languages. Cambridge: Cambridge University Press.
Mylius, K. (2015 [1983]). Historia de la literatura india antigua. Madrid: Trotta.

Oberlies, T. (2003). A grammar of Epic Sanskrit. Berlín-NY: De Gruyter.

Pollock, S. (2003). Sanskrit Literary Culture from the Inside Out. In S. Pollock (Ed.), Literary Cultures in History. Reconstructions from South Asia (pp. 39-130). Berkeley-Los Angeles: University of California Press.

Van Hall, T., \& Vielle, C. (Ed.) (2013). Grammatica Grandonica. The Sanskrit Grammar of Johann Ernst Hanxleden S.J. (1681-1732). Postdam: Universitäts Verlag Potsdam.

Witzel, M. (1989). Tracing the Vedic dialects. In C. Caillat (Ed.), Dialects dans les littératures indo-aryennes (pp. 97-264). París: College de France/Institut de Civilisation Indienne. 